\title{
Associations of neighborhood-level workplace violence with workers' mental distress problems: a multilevel analysis of Taiwanese employees
}

\author{
Li-Chung Pien ${ }^{1,2}$, Duan-Rung Chen ${ }^{1}$, Chiou-Jong CheN $^{3}$, Kuei-Min Liang ${ }^{4}$ and \\ Yawen CHENG $^{1}$
}

${ }^{1}$ Institute of Health Policy and Management, College of Public Health, National Taiwan University, Taiwan, ${ }^{2}$ Department of Nursing, Wizcare Hospital, Taiwan, ${ }^{3}$ Occupational Safety and Health Administration, Ministry of Labor, Taiwan and ${ }^{4}$ Department of Nursing, Cardinal Tien Junior College of Healthcare and Management, Taiwan

\begin{abstract}
Associations of neighborhood-level workplace violence with workers' mental distress problems: a multilevel analysis of Taiwanese employees: Li-Chung PIEN, et al. Institute of Health Policy and Management, College of Public Health, National Taiwan University, Taiwan-Objective: Workplace violence is known to pose mental health risks. However, whether or not workplace violence in a surrounding area might further increase the risk of mental distress in workers has rarely been examined. Methods: The study subjects were 9,393 male and 7,716 female employees who participated in a nationwide survey in 2010. Their personal experiences of workplace violence over the past 1 year were ascertained by a standardized questionnaire. Also assessed were their psychosocial work characteristics and mental distress problems. Neighborhood-level workplace violence was computed based on aggregated data at the county level and was categorized into low-, medium, and high-level categories. Multilevel logistic regression models were constructed to examine the associations between neighborhood-level workplace violence and individual-level mental distress problems, with adjustment of individual-level experience of workplace violence. Findings: The neighborhood-level prevalence of workplace violence ranged from 4.7 to $14.7 \%$ in men and from 6.4 to $14.8 \%$ in women across 22 counties. As compared with those who live in counties of the lowest tertile of workplace violence, female workers who lived in counties of the highest tertile of workplace violence had a 1.72-fold increased risk for mental distress problems after controlling for individual experi-
\end{abstract}

Received May 18, 2015; Accepted Aug 17, 2015

Published online in J-STAGE Sept 29, 2015

Correspondence to: Y. Cheng, Institute of Health Policy and Management, College of Public Health, National Taiwan University, 17, Xu-Zhou Rd., Taipei,Taiwan (e-mail: ycheng@ntu.edu.tw) ence of workplace violence and other psychosocial work characteristics. Conclusion: Neighborhood-level workplace violence was associated with poor mental health in female workers. Preventative strategies targeting workplace violence should pay attention to neighborhood factors and gender-specific effects that might influence societal tolerance of abusive work practices and workers' vulnerability to mental health impacts of workplace violence.

(J Occup Health 2015; 57: 555-564)

Key words: Mental health, Multi-level analysis, Workplace violence

Workplace violence is a growing mental health concern $^{1,2)}$. According to the International Labor Organization (ILO), World Health Organization (WHO), International Council of Nurses (ICN), and Public Services International (PSI), workplace violence is defined as "incidents where staff are abused, threatened or assaulted in circumstances related to their work, including commuting to and from work, involving an explicit or implicit challenge to their safety, well-being or health". Besides physical violence, violent incidents in workplace can also be psychological, such as verbal abuse, harassment, bullying/ mobbing, and threat ${ }^{3)}$.

In epidemiologic studies, workplace violence is often classified into the following four types: physical violence, verbal violence, psychological violence, and sexual harassment ${ }^{4-6}$. In a survey conducted in the United States, $0.4 \%$ of workers reported having experienced multiple forms of nonfatal workplace violence over the past year ${ }^{7)}$. In a Dutch survey, 24\% employees were found to have experienced workplace violence in the past year $^{8)}$. According to the Fifth 
European Working Conditions Survey (EWCS) of $2010,11 \%$ of workers reported having been subjected to verbal violence during the month before the survey. In the same survey, the prevalences of employees who reported being bullied, experiencing physical violence, and being sexually harassed were $4 \%, 2 \%$, and $1 \%$, respectively. Investigation in Hong Kong showed that the prevalence of workplace violence injuries increased from 1998 of 0.26 per 1,000 employees in 1998 up to 0.39 in $2007^{9}$. All these studies indicated that workplace violence is an important occupational health issue.

In addition to immediate physical harms, victims of workplace violence are also found to have greater risks for mental health problems, including anxiety, depression, stress disorders, poor sleep, sleep disorders, and sickness absence ${ }^{10-17)}$. Some studies further indicated that workplace violence could lead to serious post-traumatic stress disorder (PTSD) ${ }^{13}$. Furthermore, workplace violence has also been found to cause job stress, job dissatisfaction, work-related burnout, turnover, and intention to leave ${ }^{8,9,13)}$. Regarding work organizations, negative impacts of workplace violence on work organizations have been documented, including increased interpersonal conflicts, reduced work morale, deteriorated work performance, and increased costs associated with workers' compensation and corporate administrative expenses ${ }^{9,15,18,19)}$.

Previous studies showed that residents living in a violent neighborhood were prone to mental disorders including depression and anxiety disorders ${ }^{20)}$. While experiences of workplace violence are known to pose mental health risks in affected workers, little research has been conducted to examine whether or not working in a violent area or neighborhood where aggressive behaviors more prevalent entails additional mental health risks for workers, regardless of the workers' actual experiences of workplace violence. There are potentially multiple possible reasons for increased mental health risks when working in a violence-prone environment. First, workers who witness or hear of workplace violence might also feel threatened and anxious. Second, a high prevalence of workplace violence can be seen as an indicator of an unsafe working environment in which the safety and mental health well-being of employees are not considered as a major issue by the management. Employees working in such a social environment would be more likely to develop mental distress problems. While gender differences have been noticed in previous studies showing that women were more susceptible than man in developing mental distress when exposed to a violent living environment ${ }^{21-24)}$, to our knowledge, there has been no investigation concerning gender differences in the effects of area-level workplace violence on workers' mental health risks.

In this study, we utilized data from a national survey of the general working population in Taiwan to examine the prevalence of workplace violence by neighborhood and to examine the associations between neighborhood-level workplace violence and individuallevel mental distress status by gender, with adjustment of individual-level experience of workplace violence.

\section{Methods}

Study population and procedures

The Ministry of Labor of Taiwan has conducted nationwide surveys of the working population every 3-5 years since 1994. The subjects for the present study were participants in a cross-sectional survey conducted in September 2010. In this survey, a representative sample of employees in Taiwan was selected by a two-stage random sampling process. In the first stage, all districts and villages throughout Taiwan were grouped into 23 urbanization levels. A sample of districts and villages was randomly selected from each level. In the second stage, a random sample of households was selected within each district or village, and residents of the sampled households who were currently working at the time of the survey were identified and invited to participate in the survey.

Self-administered questionnaires were delivered to each selected households by trained interviewers. Within 1 week, the same interviewer visited the household to collect the completed questionnaires, with on-site checking. A total of 28,077 subjects were sampled, and $24,427(87 \%)$ of them agreed to participate and completed the questionnaires. The subjects included 17,286 employees and 7,141 employers and self-employed workers combined. In this study, we restricted the sample to employees who were in the age range between 25 and 65 years in consideration of the fact that working conditions of workers in this age range are stable. More information with regard to the sampling process and survey procedures can be found elsewhere ${ }^{25,26)}$.

\section{Assessment of workplace violence and mental distress problems}

With a standardized questionnaire, study participants were asked if they had ever experienced any of the following four types of workplace violence during the 12 months prior to the survey: physical violence (such as beating, kicking, pushing, pinching, pulling, etc.), verbal violence (such as abusive language, verbal harassment, cynical comments, etc.), psychological violence (such as threats, intimidation, discrimination, exclusion, bullying, harassment, etc.), and sexual harassment (such as sexually suggestive and inappropriate behavior). For each type, one item was used, 
and the answer was dichotomized into "yes" and "no" categories.

The presence of mental distress problems was assessed by one item, in which participants were asked how often they had experienced anxiety, depression, or strongly disturbed moods during the 12 months prior to the survey, and the answer was also dichotomized into "yes" and "no" categories.

\section{Compositional variables}

Compositional variables consisting of a range of work characteristics, including work shift, working hours, job control, psychological job demands, physical job demands, and employment insecurity were assessed by the questionnaire. Work shift was assessed by asking the study participant if he/she was on a fixed day shift, fixed night shift, or rotated shift during the week of the survey. The answers were categorized into two types: fixed day shift vs. night or rotated shift. Average working hours per week were measured by adding up all working hours during the week prior to the day of the survey. Psychosocial job characteristics were assessed by the Chinese version of Karasek's Job Content Questionnaire (C-JCQ), which included 3 items for job control scale (learning new things, non-repetitive work, allow own decision) and 5 items for psychological work demands (work fast, excessive work, insufficient time, concentrate on job for long time, hectic work ${ }^{27}$. The JCQ is based on the Demand-Control model, which postulates that high demands and low job control at work will lead to a higher risk of stress-related health problems. Information regarding the psychosocial properties of the C-JCQ can be found elsewhere ${ }^{28)}$. In this study, the Cronbach's $\alpha$ values for the job control scale were 0.375 for men and 0.381 for women, and those for the psychological work demands scale were 0.563 for men and 0.634 for women. Levels of workplace justice were assessed by a 7-item scale (trust employees, information reliable, work duties and responsibilities fairly arranged, benefits and welfare fairly arranged, performance fairly evaluated, information sufficient, employees being treated with respect). Its Cronbach's $\alpha$ values were 0.944 for men and 0.944 for women. Responses to the items for job control, psychological work demands, and workplace justice were recorded on a four-point Likert scale, ranging from 1 (strongly disagree) to 4 (strongly agree). The full statements, calculation formulas, and psychometric properties for job control, psychological job demands, employment security, and workplace justice can be found elsewhere ${ }^{29)}$.

In addition, there was 1 item-my job security is good-for the assessment of employment security, and 1 item-my job is physically demanding — for the assessment of physical demands. The responses to these items were dichotomized for analyses. Other information such as gender, age, educational level, and workplace (county) were also obtained by the questionnaire.

\section{Contextual variable}

Neighborhood-level of workplace violence was computed based on aggregated data by county or city. There are a total of 23 counties and cities in Taiwan, with populations ranging from 230,673 to $3,897,367$. The cities and counties were classified into low-, medium-, and high-level neighborhood violence categories based on the aggregated level of workplace violence.

\section{Statistical analysis}

We excluded subjects who did not complete items for the assessment of workplace conditions $(n=40)$. Subjects in the Penghu County were also excluded, taking into consideration that the size of this subgroup was small (84 males and 53 females) and the fact that Penghu County is a small offshore island where working conditions are dramatically different from those in Taiwan. This resulted in a final sample size of 17,109 employees (9,393 males and 7,716 females).

Descriptive statistical analyses and individual level logistic regression were performed with SAS 9.3, while multilevel logistic regression analyses were performed using HLM software version 7. All analyses were stratified by gender, taking into consideration that adverse psychosocial work factors and mental distress problems are known to differ by gender and that gender-specific factors such as coping behaviors, social roles, family burdens, and socioeconomic conditions may alter the exposure-outcome associations.

Descriptive statistics were performed to summarize the sociodemographic characteristics, mental distress problems, and work conditions of the study population. Multivariate logistic regression analyses were applied to explore the associations of the experience of workplace violence with mental distress problems. Controlled in the models were demographic and work-related variables that have been documented in previous literature to be associated with mental health outcomes. Scores for job control, psychological work demands, and workplace justice were treated as continuous variables, and other work factors such as shift work, long working hours, employment insecurity, and physical work demands were treated as dichotomized variables in logistic regression models.

Due to the hierarchical structure of the data, we employed a two-level random-effect logistic regression analysis ${ }^{30)}$ with data from individual employees (9,393 males and 7,716 females) in the first level and 
data from counties in the second level $(n=22)$. This approach allowed us to account for level variations and to estimate the variance for the parameter of interest. First, an empty model was constructed to assess whether or not the prevalence of mental distress problems among employees varied from county/city to county/city. Next, we added several compositional variables into the empty model to examine their effects on mental distress problems among employees. Finally, we used the intercept as outcome statistical model and added the contextual variable (levels of workplace violence prevalence) to the model.

\section{Results}

Table 1 displays the distribution of selected compositional variables of the studied population. The mean ages were 40.9 and 39.3 years old in men and women, respectively, and the average working hours were 43.3 and 42.8 hours per week in men and women, respectively.

The prevalence rates of workplace violence in the last 12 months in men and women were as follows: 6.9 and $7.6 \%$ for verbal violence, 3.4 and $4.1 \%$ for psychological violence, 0.8 and $0.5 \%$ for physical violence, and 0.4 and $1.7 \%$ for sexual harassment.

Table 1. Demographic characteristics, job characteristics, and prevalence of workplace violence and emotional distress in both genders $(\mathrm{N}=17,109)$

\begin{tabular}{|c|c|c|c|c|}
\hline \multirow{2}{*}{ Variable } & \multicolumn{2}{|c|}{$\operatorname{Men}(n=9,393)$} & \multicolumn{2}{|c|}{ Women $(n=7,716)$} \\
\hline & $\mathrm{n}$ & $\% / \mathrm{SD}$ & $\mathrm{n}$ & $\% / \mathrm{SD}$ \\
\hline \multicolumn{5}{|l|}{ Demographic characteristics } \\
\hline Age (years): mean (SD) & 40.9 & 10.0 & 39.3 & 9.7 \\
\hline \multicolumn{5}{|l|}{ Education level } \\
\hline Junior high and below & 1,957 & 20.8 & 1,354 & 17.6 \\
\hline Senior high & 4,746 & 50.5 & 3,866 & 50.1 \\
\hline University and above & 2,690 & 28.6 & 2,496 & 32.4 \\
\hline \multicolumn{5}{|l|}{ Work shift } \\
\hline Fixed day shift & 7,129 & 75.9 & 6,120 & 79.3 \\
\hline Night/rotated shift & 2,264 & 24.1 & 1,596 & 20.7 \\
\hline Working hours/week: mean (SD) & 43.3 & 8.9 & 42.8 & 7.9 \\
\hline$\leq 48$ hours & 8,106 & 86.3 & 6,881 & 89.2 \\
\hline$>48$ hours & 1,287 & 13.7 & 835 & 10.8 \\
\hline Job control: mean (SD) & 20.7 & 3.4 & 20.3 & 3.4 \\
\hline Missing & 27 & 0.29 & 18 & 0.23 \\
\hline Psychological work demands: mean (SD) & 12.9 & 1.8 & 12.9 & 2.0 \\
\hline Missing & 29 & 0.31 & 27 & 0.35 \\
\hline Workplace justice: mean (SD) & 19.8 & 3.7 & 19.8 & 3.8 \\
\hline \multicolumn{5}{|l|}{ Physical job demand } \\
\hline Yes & 5,409 & 57.6 & 3,459 & 44.8 \\
\hline No & 3,975 & 42.3 & 4,249 & 55.1 \\
\hline Missing & 9 & 0.09 & 8 & 0.10 \\
\hline \multicolumn{5}{|l|}{ Employment security } \\
\hline Yes & 5,025 & 53.5 & 3,955 & 51.3 \\
\hline No & 4,361 & 46.4 & 3,757 & 48.7 \\
\hline Missing & 7 & 0.07 & 4 & 0.05 \\
\hline Any type of violence (yes/no) & 757 & 8.1 & 730 & 9.5 \\
\hline Verbal violence (yes/no) & 643 & 6.9 & 582 & 7.6 \\
\hline Psychological violence (yes/no) & 321 & 3.4 & 316 & 4.1 \\
\hline Physical violence (yes/no) & 77 & 0.8 & 37 & 0.5 \\
\hline Sexual harassment (yes/no) & 36 & 0.4 & 132 & 1.7 \\
\hline Emotional distress (yes/no) & 1,814 & 19.3 & 2,137 & 27.7 \\
\hline
\end{tabular}




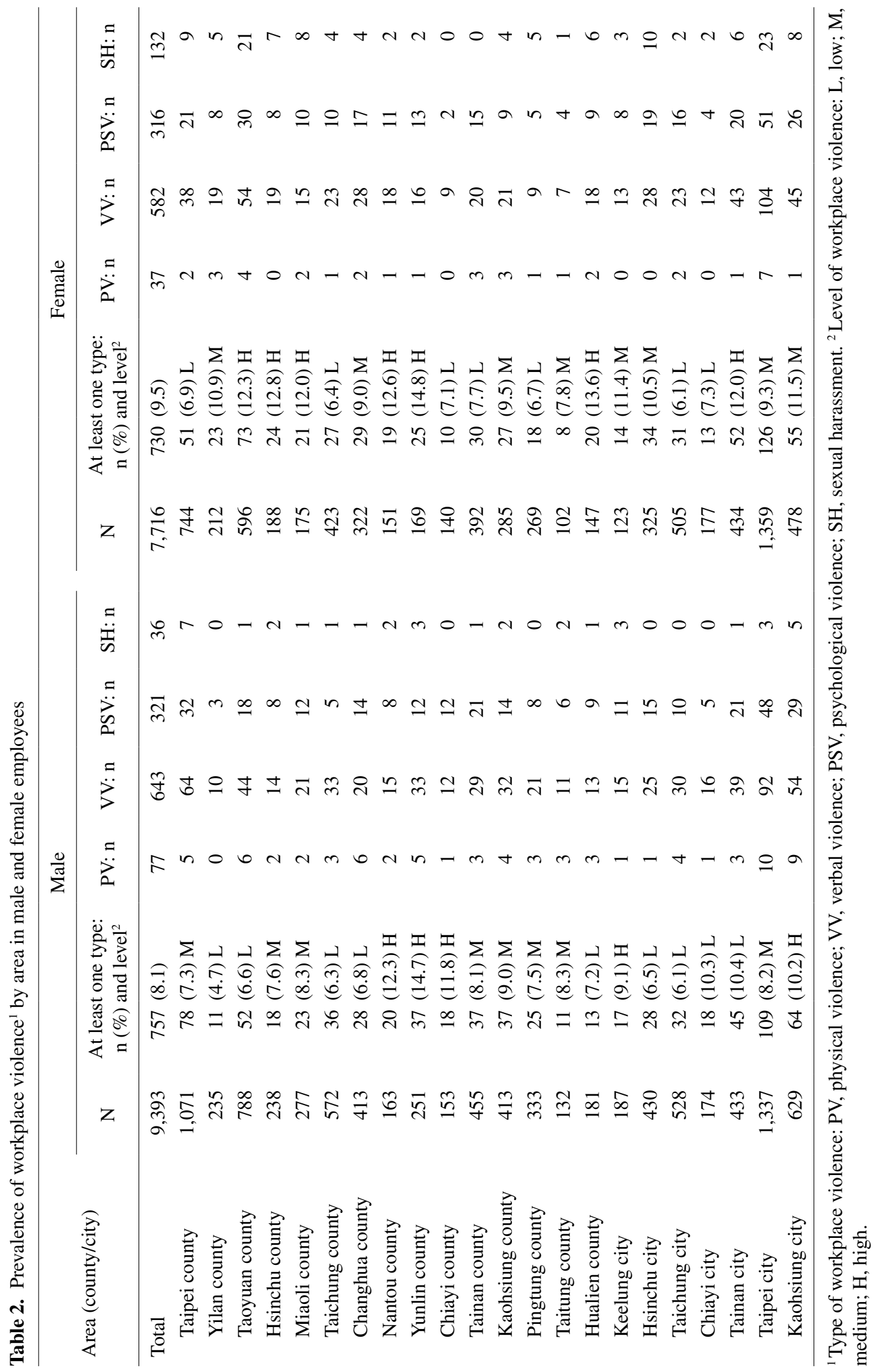


Table 3. Individual-level and county-level predictors of emotional distress: individual-level and multilevel models (male employees)

\begin{tabular}{|c|c|c|c|}
\hline & $\begin{array}{l}\text { Model with compositional } \\
\text { variables only }\end{array}$ & $\begin{array}{l}\text { Model with compositional } \\
\text { and contextual variables }\end{array}$ & $\begin{array}{l}\text { Model with compositional } \\
\text { and contextual variables }\end{array}$ \\
\hline & OR $\quad(95 \%$ CI $) \quad p$ value $^{1}$ & OR $\quad(95 \% \mathrm{CI}) \quad p$ value $^{1}$ & OR $\quad(95 \% \mathrm{CI}) \quad p$ value $^{1}$ \\
\hline \multicolumn{4}{|l|}{ Level 1} \\
\hline Intercept 1 & $0.110(0.085,0.141)^{* * *}$ & $0.125(0.097,0.161)^{* * *}$ & $0.109(0.085,0.140)^{* * *}$ \\
\hline $\begin{array}{l}\text { Individual-level workplace violence } \\
\text { (yes/no) }\end{array}$ & $3.294(2.792,3.886)^{* * *}$ & $3.311(2.805,3.908)^{* * *}$ & $3.307(2.800,3.906)^{* * *}$ \\
\hline Age $25-34$ & 1 & 1 & 1 \\
\hline Age $35-44$ & $1.316(1.209,1.432)^{* * *}$ & $1.318(1.211,1.434)^{* * *}$ & $1.317(1.210,1.433)^{* * *}$ \\
\hline Age $45-54$ & $1.395(1.270,1.532)^{* * *}$ & $1.398(1.273,1.536)^{* * *}$ & $1.397(1.272,1.534)^{* * *}$ \\
\hline Age $55-65$ & $1.053(0.838,1.323)$ & $1.055(0.841,1.324)$ & $1.053(0.839,1.323)$ \\
\hline Shift work (yes/no) & $1.138(1.056,1.226)^{* * *}$ & $1.139(1.057,1.227)^{* * *}$ & $1.139(1.057,1.227)^{* * *}$ \\
\hline Working hours $>48$ hours (yes/no) & $1.153(0.999,1.330)$ & $1.151(0.997,1.328)$ & $1.151(0.997,1.329)$ \\
\hline Job control (continuous) & $1.088(1.032,1.148)^{* *}$ & $1.089(1.032,1.148)^{* *}$ & $1.089(1.032,1.148)^{* *}$ \\
\hline $\begin{array}{l}\text { Psychological work demands } \\
\text { (continuous) }\end{array}$ & $1.252(1.214,1.290)^{* * *}$ & $1.252(1.214,1.290)^{* * *}$ & $1.252(1.214,1.290)^{* * *}$ \\
\hline Employment insecurity (yes/no) & $1.168(1.004,1.359)^{*}$ & $1.168(1.003,1.360)^{*}$ & $1.168(1.003,1.360)^{*}$ \\
\hline Physical job demands (yes/no) & $0.894(0.773,1.034)$ & $0.895(0.775,1.034)$ & $0.895(0.774,1.035)$ \\
\hline Workplace justice (continuous) & $0.930(0.912,0.948)^{* * *}$ & $0.930(0.912,0.948)^{* * *}$ & $0.930(0.912,0.948)^{* * *}$ \\
\hline Level 2 & & & \\
\hline Workplace violence low (reference) & & 1 & \\
\hline Workplace violence medium & & $0.961(0.648,1.426)$ & \\
\hline Workplace violence high & & $0.705(0.474,1.050)$ & \\
\hline $\begin{array}{l}\text { Workplace violence } \\
\text { (every } 10 \% \text { increase) }\end{array}$ & & & $0.606(0.292,1.256)$ \\
\hline Variance component & $0.151 * * *$ & $0.148 * * *$ & $0.149 * * *$ \\
\hline ICC & $0.151 /(0.151+3.29)=0.044$ & $0.148 /(0.148+3.29)=0.043$ & $0.149 /(0.149+3.29)=0.043$ \\
\hline
\end{tabular}

${ }^{1}: * p<0.05 ; * * p<0.01 ; * * * p<0.001$.

In total, $8.1 \%$ of men and $9.5 \%$ of women had been exposed to at least one of the four types of workplace violence. As shown in Table 2, there were substantial regional variations in the prevalence of any type of workplace violence across the 22 counties/cities, ranging from 4.7 to $14.7 \%$ in men and from 6.4 to $14.8 \%$ in women (Table 2).

The results of the multilevel analyses for men and women are shown in Tables 3 and 4. The intraclass correlation coefficient (ICC) was defined as the between-neighborhood variance divided by the withinneighborhood variance plus the between-neighborhood variance. Gender-specific analyses were conducted, and the ICC values of the null models were $3.5 \%$ in male subgroup and $2.9 \%$ in female subgroup, both reaching the level of statistical significance $(p<0.001)$. The results indicated that there were significant regional differences in mental distress problems. In the models that included only compositional variables, significant predictors for mental distress problems in both genders included the following: individual's experience of workplace violence, age in the range of 35-54 years old as compared with those in the age range of 25-34 years old, shift work status, low job control, high work demands, employment insecurity, and low workplace justice. It is clear that individual experience of workplace violence was the most significant predictor for mental distress problems in both men and women, with odds ratios of 3.66 and 3.25, respectively (Tables 3 and 4).

When neighborhood-level workplace violence was taken into account, noticeable reductions in statistical model variance components (in men from 0.154 to 0.138 and in women from 0.104 to 0.045 ) and in ICCs (in men from 0.045 to 0.040 and in women from 0.031 to 0.013) were found, indicating that neighborhood-level workplace violence contributed to the risk of mental distress problems. In particular, female workers who 
Table 4. Individual-level and county-level predictors of emotional distress: individual-level and multilevel models (female)

\begin{tabular}{|c|c|c|c|}
\hline & $\begin{array}{l}\text { Model with compositional } \\
\text { variables only }\end{array}$ & $\begin{array}{l}\text { Model with compositional } \\
\text { and contextual variables }\end{array}$ & $\begin{array}{l}\text { Model with compositional } \\
\text { and contextual variables }\end{array}$ \\
\hline & OR $\quad(95 \%$ CI $) p$ value $^{1}$ & OR $\quad(95 \%$ CI $) p$ value $^{1}$ & OR $\quad(95 \%$ CI $) p$ value $^{1}$ \\
\hline \multicolumn{4}{|l|}{ Level 1} \\
\hline Intercept 1 & $0.264(0.166,0.418)^{* * *}$ & $0.185(0.121,0.284)^{* * *}$ & $0.265(0.174,0.403)^{* * *}$ \\
\hline $\begin{array}{l}\text { Individual-level workplace violence } \\
\text { (yes/no) }\end{array}$ & $2.945(2.400,3.614)^{* * *}$ & $2.921(2.378,3.588)^{* * *}$ & $2.924(2.381,3.592) * * *$ \\
\hline Age $25-34$ & 1 & & 1 \\
\hline Age $35-44$ & $1.178(1.027,1.352)^{*}$ & $1.179(1.027,1.353)^{*}$ & $1.178(1.027,1.352)^{*}$ \\
\hline Age $45-54$ & $0.970(0.842,1.117)$ & $0.969(0.840,1.118)$ & $0.968(0.839,1.116)$ \\
\hline Age $55-65$ & $0.839(0.631,1.116)$ & $0.841(0.632,1.119)$ & $0.840(0.631,1.118)$ \\
\hline Shift work (yes/no) & $1.155(1.030,1.296)^{*}$ & $1.155(1.028,1.297)^{*}$ & $1.154(1.028,1.294)^{*}$ \\
\hline Working hours $>48$ hours (yes/no) & $0.979(0.736,1.303)$ & $0.978(0.734,1.304)$ & $0.980(0.736,1.305)$ \\
\hline Job control (continuous) & $1.096(1.052,1.142)^{* * *}$ & $1.096(1.052,1.142)^{* * *}$ & $1.096(1.052,1.142)^{* * *}$ \\
\hline $\begin{array}{l}\text { Psychological work demands } \\
\text { (continuous) }\end{array}$ & $1.255(1.216,1.297)^{* * *}$ & $1.256(1.215,1.297)^{* * *}$ & $1.256(1.215,1.297)^{* * *}$ \\
\hline Employment insecurity (yes/no) & $1.014(0.904,1.137)$ & $1.015(0.905,1.138)$ & $1.015(0.905,1.139)$ \\
\hline Physical job demands (yes/no) & $1.012(0.886,1.155)$ & $1.013(0.887,1.156)$ & $1.012(0.886,1.156)$ \\
\hline Workplace justice (continuous) & $0.930(0.914,0.946)^{* * *}$ & $0.930(0.914,0.946)^{* * *}$ & $0.930(0.914,0.946)^{* * *}$ \\
\hline \multicolumn{4}{|l|}{ Level 2} \\
\hline Workplace violence low (reference) & & 1 & \\
\hline Workplace violence medium & & $1.636(1.276,2.098)^{* * *}$ & \\
\hline Workplace violence high & & $1.749(1.270,2.410)^{* *}$ & \\
\hline $\begin{array}{l}\text { Workplace violence } \\
\text { (every } 10 \% \text { increase) }\end{array}$ & & & $2.154(1.123,4.132)^{*}$ \\
\hline Variance component & $0.108 * *$ & $0.047 * *$ & $0.068 * *$ \\
\hline ICC & $0.108 /(0.108+3.29)=0.032$ & $0.047 /(0.047+3.29)=0.014$ & $0.068 /(0.068+3.29)=0.020$ \\
\hline
\end{tabular}

${ }^{1}: * p<0.05 ; * * p<0.01 ; * * * p<0.001$.

lived in counties of the highest tertile of workplace violence had a 1.72-fold increased risk of mental distress problems as compared with those who lived in counties of the lowest tertile of workplace violence, after controlling for individual experience of workplace violence and other work characteristics. However, no association was found in men.

\section{Discussion}

While it is known that the experience of workplace violence constitutes a mental health risk in affected workers $^{10,13,15-17,31-34)}$, the results of the present study further suggest that a higher level of neighborhoodlevel workplace violence was associated with an additional increased risk of mental distress problems in female workers but not male workers. Gender differences may be due to a greater susceptibility of women to violent external environment as reported in previous studies $^{21-24)}$.

The findings of this study suggest that the preva- lence of workplace violence in Taiwan was not as high as in western societies. Nevertheless, direct comparisons of the level of workplace violence across countries and studies should be made with caution, because workplace violence might be defined and assessed quite differently. Furthermore, social awareness of workplace violence, which varies across cultures and societies, may also affect how this problem is defined and reported ${ }^{4,5)}$. Especially for the types of verbal violence, psychological violence and sexual harassment, the measures would be subject to great variations due to social and cultural factors. However, in terms of the types of workplace violence, verbal violence and psychological violence were more prevalent than other types, which was consistent with findings from western countries ${ }^{5,9,18)}$. The reason for a higher risk of workplace violence in women than in men, as found in previous studies, is perhaps due to the disadvantaged working and socioeconomic conditions among female workers, which render them 
relatively lower levels of social resources ${ }^{35,36)}$. With regard to the uneven distribution in the levels of workplace violence across the occupational spectrum and regions, differences in industry structure, work conditions, and work cultures could be the causes of the differences ${ }^{4}$.

There were some limitations in this study. First, the nature of the cross-sectional study design precluded direct casual interpretation between workplace violence and the risk of mental health problems. Reverse causality cannot be ruled out. For instance, employees with mental health problems may be more vulnerable to becoming victims of workplace violence. In addition, there is a possibility that workers with a poor mental health status may over-interpret or overestimate the problems of workplace violence. Future research should adopt a longitudinal study design to examine how the experience of workplace violence may deteriorate workers' mental health status, taking into account workers' personality traits or other confounders.

Second, the assessment of workplace violence in this study was rough and based on self-report. Because no further explanations were given and participants may perceive or interpret workplace violence differently, the validity of these measures may be questionable. Furthermore, despite the fact that four types of workplace violence were specified, the severity, frequency, and sources of workplace violence were not assessed. In contrast, studies from other countries such as the EU and the U.S. distinguished workplace violence arising inside the work organization (including supervisors and colleagues) from that arising outside the work organization (including clients, customers, and strangers $)^{5,9,37)}$. The perception of workplace violence, especially for the types of psychological violence and sexual harassment, may also be influenced by respondents' subjective attitudes, which will also affect whether individuals evaluate problems as stressful or not ${ }^{14)}$. Improvement in the measurement and classification of workplace violence as well as assessment of individual perceptions will be essential for the identification of leveraging points for interventions. We recommend that future research should obtain information concerning the sources, frequency, severity, and nature of workplace violence in a more objective manner, as well as information concerning participants' attitudes and perceptions toward different types of workplace violence.

Third, although potential confounding factors including age, shift work status, working hours, and work-related characteristics were controlled in our statistical models, there are still other potential workrelated factors and individual characteristics (such as socioeconomic status, occupational status, social support, or resources) ${ }^{38-40)}$ that could confound or modify the associations between workplace violence and mental health status of workers. Gender differences in the distribution of uncontrolled confounders might explain why a higher level of neighborhoodlevel workplace violence was associated with an additional risk of mental distress among female workers but not male workers.

Furthermore, neighborhood-level workplace violence was defined by aggregated data based on the administrative unit of "city or county", which may not reflect participants' real-life experiences in the community. Finally, the Cronbach's alpha value for the internal consistency of the measures for job control and psychological work demands in this study were low, because only a limited number of items were used.

Despite of the abovementioned limitations, the results of this study are still important because they indicate that working in a neighborhood where workplace violence is prevalent would increase the risk of mental health problems in female workers after controlling for their actual experiences of workplace violence. We suggest that preventative strategies targeting workplace violence should pay attention to neighborhood factors as well as gender-specific effects that might influence societal tolerance to abusive work practices and workers' vulnerability to mental health impacts of workplace violence.

Acknowledgment: This study was financially supported by a research grant from the Ministry of Science of Taiwan (NSC 102-2410-H-002 -071 -MY2). The authors declare that they have no conflicts of interest.

\section{References}

1) Estrada F, Nilsson A, Jerre K, Wikman S. Violence at work-The emergence of a social problem. J Scand Stud Criminol Crime Prev 2010; 11: 46-65.

2) Pilch I, Turska E. Relationships between machiavellianism, organizational culture, and workplace bullying: emotional abuse from the Target's and the perpetrator's perspective. J Bus Ethics 2014: 1-11.

3) Martino Vd. Workplace violence in the health sector: country case studies: Brazil, Bulgaria, Lebanon, Portugal, South Africa, Thailand, and an additional Australian study. Geneva, Switzerland: ILO/ICN/WHO/PSI Joint Programme on Workplace Violence in the Health Sector. 2002.

4) Niedhammer I, Sultan-Taieb H, Chastang JF, Vermeylen G, Parent-Thirion A. Exposure to psychosocial work factors in 31 European countries. Occup Med (Oxf) 2012; 62: 196-202.

5) Parent-Thirion A, Vermeylen G, van Houten G, Lyly-Yrjänäinen M, Biletta I, Cabrita J. Fifth European working conditions survey-Overview report 2012: 57-9. 
6) Lin MX, Guo ZY. Survey of Perceptions of Safety and Health in the Work Environment in 2013 Taiwan. Annual Research Report of Institute of Occupational Safety and Health, Council of Labor Affairs, ROC (Taiwan), 2013 Project Number IOSH102-M306 Taipei: Institute of Occupational Safety and Health, Council of Labor Affairs, Executive Yuan, ROC (Taiwan), 2013 [In Chinese: English abstract]. 2013.

7) Harrell E. Workplace Violence, 1993-2009. National Crime Victimization Survey and the Census of Fatal Occupational Injuries Washington, DC: Bureau of Justice Statistics. [Online]. 2011 [cited 2014 Dec 25]: Available from: URL: wwwbjsgov/indexcfm?ty $=$ pbdetail\&iid $=2377$

8) Venema A, Klauw Mvd. Impact of Workplace Violence on Employees. European Working Condictions Observatory, EWCO. [Online]. 2012 [cited 2014 Dec 27]: Available from: URL: http:// eurofoundeuropaeu/observatories/eurwork/articles/ other/impact-of-workplace-violence-on-employees

9) Lo TW, Chappell D, Kwok SI, Wu J. Workplace violence in Hong Kong, China: nature, impact, and preparedness. Int J Offender Ther 2012; 56: 955-75.

10) Takaki J, Taniguchi T, Fukuoka E, et al. Workplace bullying could play important roles in the relationships between job strain and symptoms of depression and sleep disturbance. J Occup Health 2010; 52: 367-74.

11) Lallukka $T$, Rahkonen O, Lahelma E. Workplace bullying and subsequent sleep problems-the Helsinki Health Study. Scand J Work Environ Health 2011; 37 : 204-12.

12) Christiansen RH, Nielsen HO. Negative health outcomes resulting from bullying in the workplace. European Working Conditions Observatory (EWCO). [Online]. 2010 [cited 2015 Jan 20]: Available from: URL: http://www.eurofound.europa.eu/observatories/ eurwork/articles/working-conditions/negative-healthoutcomes-resulting-from-bullying-in-the-workplace

13) Willness CR. A Meta-analysis of the antecedents and consequences of workplace sextual harassment. J Pers Psychol 2007; 60: 127.

14) Vie TL, Glaso L, Einarsen S. Health outcomes and self-labeling as a victim of workplace bullying. J Psychosom Res 2011; 70: 37-43.

15) Hansen AM, Hogh A, Persson R, Karlson B, Garde $\mathrm{AH}$, Orbaek P. Bullying at work, health outcomes, and physiological stress response. J Psychosom Res 2006; 60: 63-72.

16) Mayhew C, Chappell D. Workplace violence: an overview of patterns of risk and the emotional/stress consequences on targets. Int J Law Psychiat 2007; 30: 327-39.

17) Aytac S, Bozkurt V, Bayram N, et al. Workplace violence: a study of Turkish workers. Int J Occup Saf Ergo 2011; 17: 385-402.

18) Demir D, Rodwell J. Psychosocial antecedents and consequences of workplace aggression for hospital nurses. J Nurs Scholarship 2012; 44: 376-84.
19) Speroni KG, Fitch T, Dawson E, Dugan L, Atherton $\mathrm{M}$. Incidence and cost of nurse workplace violence perpetrated by hospital patients or patient visitors. J Emerg Nurs 2014; 40: 218-28.

20) Clark C, Ryan L, Kawachi I, Canner MJ, Berkman L, Wright RJ. Witnessing community violence in residential neighborhoods: a mental health hazard for urban women. J Urban Health 2008; 85: 22-38.

21) Mausner-Dorsch H, Eaton WW. Psychosocial work environment and depression: epidemiologic assessment of the demand-control model. Am J Public Health 2000; 90: 1765.

22) Okurame DE. Impact of negative workplace factors and the moderator effects of gender on critical organisational climate for effectiveness. Gender Behav 2013; 11: 5618-34.

23) Javdani S, Abdul-Adil J, Suarez L, Nichols SR, Farmer AD. Gender differences in the effects of community violence on mental health outcomes in a sample of low-income youth receiving psychiatric care. Am J Commun Psychol 2014; 53: 235-48.

24) Kaminer D, Hardy A, Heath K, Mosdell J, Bawa $\mathrm{U}$. Gender patterns in the contribution of different types of violence to posttraumatic stress symptoms among South African urban youth. Child Abuse Neglect 2013; 37: 320-30.

25) Cheng Y, Chen C-W, Chen C-J, Chiang T-1. Job insecurity and its association with health among employees in the Taiwanese general population. Soc Sci Med 2005; 61: 41-52.

26) Yeh W-Y, Cheng Y, Chen C-J. Social patterns of pay systems and their associations with psychosocial job characteristics and burnout among paid employees in Taiwan. Soc Sci Med 2009; 68: 1407-15.

27) Karasek R, Brisson C, Kawakami N, Houtman I, Bongers P, Amick B. The Job Content Questionnaire (JCQ): an instrument for internationally comparative assessments of psychosocial job characteristics. J Occup Health Psychol 1998; 3: 322-55.

28) Cheng Y, Luh WM, Guo YL. Reliability and validity of the Chinese version of the Job Content Questionnaire in Taiwanese workers. Int $\mathrm{J}$ Behav Med 2003; 10: 15-30.

29) Cheng Y, Huang H-Y, Li P-R, Hsu J-H. Employment insecurity, workplace justice and employees' burnout in Taiwanese employees: a validation study. Int J Behav Med 2011; 18: 391-401.

30) Merlo J, Chaix B, Ohlsson H, et al. A brief conceptual tutorial of multilevel analysis in social epidemiology: using measures of clustering in multilevel logistic regression to investigate contextual phenomena. J Epidemiol Commun H 2006; 60: 290-7.

31) Niedhammer I, David S, Degioanni S, et al. Workplace bullying and psychotropic drug use: the mediating role of physical and mental health status. Ann of Occup Hyg 2011; 55: 152-63.

32) Couto MT, Lawoko S, Svanstrom L. Exposure to workplace violence and quality of life among drivers and conductors in Maputo city, Mozambique. Int J Occup Environ Health 2009; 15: 299-304. 
33) Choi ES, Jung HS, Kim SH, Park H. [The influence of workplace violence on work-related anxiety and depression experience among Korean employees]. J Korean Acad Nurs 2010; 40: 650-61.

34) Pien L-C, Cheng Y-W, Chen I-S, Chen C-J. The prevalence of workplace violence and its association with the health status of Taiwanese employees. Taiwan J Public Health 2014; 33: 36-50.

35) Lawoko S, Soares JJ, Nolan P. Violence towards psychiatric staff: a comparison of gender, job and environmental characteristics in England and Sweden. Work Stress 2004; 18: 39-55.

36) Kamchuchat C, Chongsuvivatwong V, Oncheunjit S, Yip TW, Sangthong R. Workplace violence directed at nursing staff at a general hospital in southern Thailand. J Occup Health 2008; 50: 201-7.
37) Bureau of Labor Statistics DoL. Survey of Workplace Violence Prevention, 2005. Survey of Workplace Violence Prevention. [Online]. 2006 [cited 2014 Dec 15]: Available from: URL: http://www.bls. gov/iif/oshwc/osnr0026.pdf

38) Tsuno K, Kawakami N, Tsutsumi A, et al. Socioeconomic determinants of bullying in the workplace: a national representative sample in Japan. PLoS One 2015; 10: e0119435.

39) Sakurai K, Kawakami N, Yamaoka K, Ishikawa H, Hashimoto $\mathrm{H}$. The impact of subjective and objective social status on psychological distress among men and women in Japan. Soc Sci Med 2010; 70: 1832-9.

40) Virtanen M. Work characteristics, anxiety and depression. Occup Environ Med 2008; 65: 71. 